\title{
Suboptimal Frequency-Selective Transceiver Design for Multicarrier Millimeter Wave MIMO Systems
}

\author{
Nihat Kabaoğlu \\ Department of Electrical and Electronics Engineering, İstanbul Medeniyet University School of Engineering and Natural Science, İstanbul, Turkey
}

Cite this article as: N. Kabaoğlu. "Suboptimal Frequency-Selective Transceiver Design for Multicarrier Millimeter Wave MIMO Systems". Electrica, vol. 18, no. 1, pp. 83-89, 2018.

\begin{abstract}
Fifth-generation (5G) cellular communication systems aim to obtain a higher data rate, decreased latency time, higher performance even at high mobility speeds, decreased system complexity, lower transmission cost, and an increased system capacity and coverage area. Many of these goals can be achieved because of the studies devoted to the physical layer of $5 \mathrm{G}$ cellular networks. In this respect, solutions to the problems of beamforming (steering and precoding or precoding and combining) and channel estimation that are encountered in the physical layer of $5 \mathrm{G}$ cellular networks are the key points to achieve the aforementioned goals. Thus, a two-stage beamforming method is proposed in this study. The proposed method is a suboptimal method that minimizes the difference between outputs obtained when fully digital and hybrid beamforming methods are used. The analytical results, which are validated through simulations, demonstrate that the proposed method is an effective solution and, hence, the preferred beamforming approach for $5 \mathrm{G}$ millimeter wave band-based wireless systems.
\end{abstract}

Keywords: Millimeter wave communications, hybrid beamforming, precoding and combining

Address for Correspondence:

Nihat Kabaoğlu

E-mail:

nihat.kabaoglu@medeniyet.edu.tr

Received: 16.11 .2017

Accepted: 05.12 .2017

(c) Copyright 2018 by Electrica

Available online at

http://dergipark.gov.tr/iujeee

DOI: 10.5152/iujeee.2018.1813

\section{Introduction}

With intelligent devices taking an important role in our lives, demand for high data rate and system capacity is increasing day by day. The Long Term Evolution (LTE) standard, which is the standard of today's fourth-generation (4G) systems, has been able to meet these demands to a certain extent by using Orthogonal Frequency Division Multiplexing (OFDM) and Multiple-Input Multiple-Output (MIMO) technology on a network designed from the outset. However, with the extraordinary demand for applications that require more bandwidth, such as very high resolution video applications, the contradiction between capacity requirements and spectrum constraints has become more apparent. It is not possible to meet this demand with existing cellular network technology, which has a very limited bandwidth and a fairly crowded spectrum. Due to the aforementioned bandwidth bottleneck, research has been initiated to provide innovative solutions for $5 \mathrm{G}$ celIular communication systems to support the total mobile user network traffic expected to increase 1000 times by 2020 [1]. Millimeter wave (mmW) communication for physical layers of personal Wireless Area Networks (WPAN), Local Area Networks (LAN) and Metropolitan Area Networks (MAN) has already been standardized [2,3] and has a data rate potential of Gbits at the moment, $\mathrm{mmW}$ communication has also become a strong candidate for $5 \mathrm{G}$ cellular networks.

The $\mathrm{mmW}$ band is the frequency range defined as $30 \mathrm{GHz}-300 \mathrm{GHz}$ ( $3 \mathrm{GHz}$ to $30 \mathrm{GHz}$ according to some studies). There are several advantages to be gained by using $\mathrm{mmW}$ band in $5 \mathrm{G}$ cellular networks. The first one of them is that the $\mathrm{mmW}$ band provides a wide spectrum. The other is that the communication in this band is short-range one due to the severe path loss, and thus it is possible to reuse the frequency at short distances and hence it is possible to extremely increase the capacity. Another is that very high data rates can be achieved by communicating with antenna arrays consisting of several 
antennas since antenna dimensions are very small in this band. The last one is that higher levels of privacy and security can be achieved if the beam width of the antenna arrays can be narrowed sufficiently. Since all these points strongly indicate that $\mathrm{mmW}$ band communication can be used in $5 \mathrm{G}$ cellular networks, studies on the channel characteristics of $\mathrm{mmW}$ band and the study of device technologies that can work on this band have started to take place in the literature in recent years $[4,5]$.

Since signal propagation in the $\mathrm{mmW}$ band has a different characteristic than the propagation in the traditional band, the vast majority of the published work is on the physical layer of $\mathrm{mmW}$ systems. These studies are usually focused on beamforming and/or channel estimation. In particular, beamforming is crucial for achieving a reasonable link budget and for overcoming high path loss problem in cellular mmW systems. It is possible to achieve higher gain because the $\mathrm{mmW}$ frequencies have the ability to fit several tens of antennas into small volumes. However, it is almost impossible to allocate a Radio Frequency (RF) chain (consisting of amplifier, mixer, analog-to-digital converter and analogue converter units) for each antenna element in this array due to the high cost and power consumption that will arise. Therefore, it is not possible to use digital beamforming methods. For this reason, the past studies proposed analog beamforming methods that operate according to the principle of controlling the phase of the signal radiated from each antenna by using RF phase shifter circuitry $[2,6,7]$. These solutions are based on the principle of recursively obtaining the beamformer coefficients in systems that do not have channel information. Similar to $[2,6]$ proposed a dual beam-training algorithm that selects a beam pattern from a codebook. In order to minimize duration of beam-training, a multi-beam structure was used in [8]. The methods proposed in these studies tend to converge to a single communication beam, despite the reduction of complexity in [2]. Hence, they are not capable of providing the desired multiplexing gain in parallel transmission of multiple data sequences. In summary, the solutions presented in the studies on analogue beamforming are not adequate due to hardware limitations.

Hybrid beamforming solutions are presented to enable higher data rate and antenna array gain [9-13]. [9] presents a hybrid beamforming solution that maximizes channel capacity, while matching one or more RF beam pairs for predefined RF beam clusters in the receiver and transmitter. The performance of the proposed method in that work is based on the diversity of the codebook for each selected RF spectrum pair since it determines the best precoder by exploring the codebook in detail (which results a high computational burden). Moreover, it is inevitable that the method continuously needs feedback information. Assuming that the receiver knows the channel perfectly, [10] proposes a simple, hybrid beamforming method that uses $\mathrm{mmW}$ channel sparsity. Under the assumption that the receiver partly knows the channel, a hybrid beamforming method is described in [11] similar to [10]. All of the methods proposed in these studies require the channel estimation and beamforming operations to be done together, since they need partial or complete channel state information during determination of the precoding matrix. Therefore, a fairly high computational burden occurs at the channel estimation stage of these proposed methods, which prevents their practical implementation. Since MIMO techniques causes a trade-off between multiplexing gain and diversity, studies on application of hybrid beamforming methods to them has been proposed in recent years. In this context, in [12], it is shown that spatial multiplexing and beamforming can be executed one after the other in actual channel models. [13] discusses the advantages and disadvantages of hybrid beamforming and low resolution combining that are two possible MIMO mmW architectures. As a result, analogue beamforming is exploited to avoid path loss in short distance communications; the hybrid beamforming is exploited to balance the trade-off between performance and complexity for new generation outdoor cellular networks.

In this work, we propose a suboptimal frequency selective transceiver design that configures the beam via the two-stage serial optimization method unlike other transceiver designs in the literature that jointly configure the beam in both Base Station (BS) and Mobile Station (MS) sides. Thus, the computational complexity of the classic transceiver algorithm is reduced by transforming the multi-parametric and multi-dimensional optimization problem into fewer parametric and lower dimensional two-parallel optimization problems.

\section{System Model}

The considered system in this work is a $\mathrm{mmW}$ MIMO-OFDM system. It is assumed that transmitter BS and receiver MS communicates each other with $N_{S}$ length- $M$ data symbol blocks over frequency selective fading channels via their one dimensionally antenna arrays. Transmitter and receiver have $N_{B S}$ and $N_{M S}$ antenna elements respectively, and $N_{R F}$ RF chains. The transmitted symbol vector $\mathbf{s}(m) \in C^{N_{S}}$ on the $m$ sub-carrier of the BS has zero-mean and $\mathbb{E}\left[\mathbf{s}(m) \mathbf{s}(m)^{H}\right]=\left(P_{t} / M N_{s}\right) \mathbf{I}_{N_{s}}$, where $P_{t}$ is the average total transmit power. $\mathbf{s}(m)$ at each sub-carrier is precoded by digital precoding matrix $\mathbf{P}_{B B}(m) \in C^{N_{R F} \times N_{S}}$, and then the resulting symbol blocks are transformed into time domain using $N_{R F} \quad M$-point IFFT assuming that all sub-carriers are used and data blocks length is equal to number of sub-carriers. After adding cyclic prefix and applying RF precoding by $\mathbf{P}_{R F} \in C^{N_{B S} \times N_{R F}}$ each element of which has constant module, we finally obtain the following discrete-time complex baseband signal on the $m$-th sub-carrier

$$
\mathbf{x}(m)=\mathbf{P}_{R F} \mathbf{P}_{B B}(m) \mathbf{s}(m)
$$

The signal in (1) is transmitted to the MS by the BS through frequency selective quasi-static channel whose discrete impulse response on $m$-th sub-carrier is $\mathbf{H}(m) \in C^{N_{M S} \times N_{B S}}$ and the 
maximum excess delay is the same as length of a symbol. After combining the received signal by $\mathbf{C}_{R F} \in C^{N_{M S} \times N_{R F}}$ and removing cyclic prefix from it, the resulting signals are transformed back to frequency domain using $N_{R F} M$-point FFT first, and then obtained symbols on each sub-carrier are combined by digital combining matrix $\mathbf{C}_{B B}(m) \in C^{N_{R F} \times N_{S}}$. The received signal on sub-carrier $m$ can be eventually obtained as

$$
\begin{aligned}
\mathbf{r}(m) & =\mathbf{C}_{B B}^{H}(m) \mathbf{C}_{R F}^{H} \mathbf{H}(m)(\mathbf{x}(m)+\mathbf{w}(m)) \\
& =\mathbf{C}_{B B}^{H}(m) \mathbf{C}_{R F}^{H} \mathbf{H}(m) \mathbf{P}_{R F} \mathbf{P}_{B B}(m) \mathbf{s}(m) \\
& +\mathbf{C}_{B B}^{H}(m) \mathbf{C}_{R F}^{H} \mathbf{w}(m),
\end{aligned}
$$

where $\mathbf{w}(m) \sim \mathcal{N}\left(\mathbf{0}, \sigma_{w}^{2} \mathbf{I}_{N_{\text {MS }}}\right)$ is the zero mean circularly symmetric complex Gaussian noise.

\section{Problem Formulation}

The aim of this work proposes a low computationally complex hybrid beamforming method for the system model given in Section 2, which maximizes the achievable rate subject to the transmit power constraint at BS side. Assuming that the mobile station is using the optimal nearest decoding method with fully digital hardware, it is possible to decouple the problems of designing beamforming and combining matrices from each other. This makes it possible to design a hybrid precoder that will maximize the common information of the system. The classic solution to this problem is given below.

$$
\begin{aligned}
\max _{\mathbf{P}_{R F}, \mathbf{P}_{B B}(m), \mathbf{C}_{R F}, \mathbf{C}_{B B}(m)} & \mathcal{I}\left(\mathbf{P}_{R F},\left\{\mathbf{P}_{B B}(m)\right\}_{m=1}^{M}, \mathbf{C}_{R F},\left\{\mathbf{C}_{B B}(m)\right\}_{m=1}^{M}\right) \\
\text { s.t. } & \mathbf{P}_{R F} \in \mathcal{P}_{R F} \text { and } \mathbf{C}_{R F} \in \mathcal{C}_{R F} \\
& \sum_{m=1}^{M}\left\|\mathbf{P}_{B B}(m)\right\|_{F}^{2} \leq P_{t}
\end{aligned}
$$

where $\mathcal{P}_{R F}$ and $\mathcal{C}_{R F}$ are general set of matrices with constant modulus entries or the quantized codebooks, and

$$
\begin{array}{r}
\mathcal{I}\left(\mathbf{P}_{R F},\left\{\mathbf{P}_{B B}(m)\right\}_{m=1}^{M}, \mathbf{C}_{R F},\left\{\mathbf{C}_{B B}(m)\right\}_{m=1}^{M}\right)=\log 2 \operatorname{det}\left(\mathbf{I}_{N_{M S}}\right. \\
\left.+\frac{\gamma}{N_{s}}\left(\mathbf{C}_{R F} \mathbf{C}_{B B}(m)\right)^{H} \mathbf{H}(m) \mathbf{R H}(m)^{H} \mathbf{C}_{R F} \mathbf{C}_{B B}(m)\right)
\end{array}
$$

is the mutual information of the system, with $\gamma=\frac{P_{t}}{M \sigma^{2}}$ and $\mathbf{R}=\mathbf{P}_{R F} \mathbf{P}_{B B}(m)\left(\mathbf{P}_{R F} \mathbf{P}_{B B}(m)\right)^{H}$. The problem in (3) is a non-convex and it needs multiparameters and multi-dimensional non-convex optimization, but it has a closed form solution.

\section{Channel Model}

In $\mathrm{mmW}$ band, free-space propagation path loss is extremely higher than traditional microwave band. Since $\mathrm{mmW}$ propagation enviorement is well characterized by a clustered channel model, mmW channel model at almostly all works in the literature such as [3,14-20] is based on Saleh-Valenzualle geometric channel model in [21]. Since the author believes that the best representation among them is the representation in [20], he adopt the same channel model with $L$ clusters, each of which has a time delay $\tau_{l} \in R$, and Angle of Arrival (AoA) and Angle of Departure (AoD) $\theta_{l}, \phi_{l} \in[0,2 \pi]$. Each ray has a relative time delay $\tau_{r_{l}}$, relative angle of arrival and departure shift $\psi_{r_{l}}, \varphi_{r_{l}}$ , and complex path gain $\alpha_{r_{l}}$. The number of rays and path loss between BS and MS are $R_{l}$ and $\rho$ respectively. $p_{R C}(\tau)$ represents raised-cosine pulse shaping function for $T_{s}$-spaced signaling evaluated at $\tau$ seconds. Delay- $d$ MIMO channel matrix $\mathbf{H}(d)$ can be expressed as [15]

$$
\begin{array}{r}
\mathbf{H}(d)=\sqrt{\frac{N_{B S} N_{M S}}{\rho}} \sum_{l=1}^{L} \sum_{r_{l}=1}^{R_{l}} \alpha_{r_{l}} p_{R C}\left(d T_{s}-\tau_{l}-\tau_{r_{l}}\right) \\
\times \mathbf{a}_{M S}\left(\theta_{l}-\psi_{r_{l}}\right) \mathbf{a}_{B S}^{H}\left(\phi_{l}-\varphi_{r_{l}}\right),
\end{array}
$$

where $\mathbf{a}_{M S}(\theta)$ and $\mathbf{a}_{B S}(\phi)$ are antenna array response vetors of MS and BS, respectively. Given the delay- $d$ MIMO channel model in (5), the channel at sub-carrier $m, \mathbf{H}(m)$ can be written as [22],

$\mathbf{H}(m)=\sum_{d=0}^{D-1} \mathbf{H}(d) \exp \left(-j \frac{2 \pi m}{M} d\right)$.

\section{Proposed Suboptimal Transceiver Design}

Nearly all previous work on hybrid beamforming for $\mathrm{mmW}$ systems uses block diagonalization method such as [23-27] since it has low complexity. Thus, a hybrid beamforming method based on the block diagonalization technique is proposed in this study. The proposed method is based on the finding of the hybrid beamformer matrices that make the received signal $\mathbf{r}(m)$ be the nearest to the signal $\mathbf{r}_{d}(m)$ obtained using digital beamformer matrices. As a metric of this closeness, the way to minimize the Mean Square Error (MSE) between the received signal $\mathbf{r}(m)$ and the signal $\mathbf{r}_{d}(m)$ to be received if a digital beamformer was used, was chosen. In this context, we calculate the MSE between $\mathbf{r}_{d}(m)$ and $\mathbf{r}(m)$ as follows:

$\mathbf{r}_{d}(m)=\mathbf{s}(m)+\mathbf{U}^{H}(m) \mathbf{w}(m)$

where $\quad \mathbf{r}_{d}(m)=\mathbf{C}_{o p t}^{H}(m) \mathbf{H}(m) \mathbf{P}_{o p t}(m) \mathbf{s}(m)+\mathbf{C}_{o p t}^{H}(m) \mathbf{w}(m)$ with both fully digital combiner matrix $\mathbf{C}_{o p t}$ and precoding matrix $\mathbf{P}_{\text {opt }}$. Minimizing $e_{m}$ for $\forall m$ with respect to parameter set $\left\{\mathbf{P}_{R F}, \mathbf{P}_{B B}(m), \mathbf{C}_{R F}, \mathbf{C}_{B B}(m)\right\}$ under the constraints in (3) gives optimum hybrid precoder matrices $\mathbf{P}_{R F}$ and $\mathbf{P}_{B B}(m)$, and combiner matrices $\mathbf{C}_{R F}$ and $\mathbf{C}_{B B}(m)$, but it still requires needs $M$ -dimensional non-convex optimization such as in [19] and [20]. However, this problem can be more easily solved when channel state information is available in MS side (this assumption is a reasonable, since channel reciprocity can be assumed when BS communicates to MS in time division duplexing mode). In that case, $\mathbf{r}_{d}(m)$ can be expressed as

$\mathbf{r}_{d}(m)=\mathbf{s}(m)+\mathbf{U}^{H}(m) \mathbf{w}(m)$,

where $\mathbf{C}_{o p t}(m)=\mathbf{U}(m)$ and $\mathbf{P}_{o p t}(m)=\mathbf{V}(m) \square(m)$, and $\mathbf{U}(m)$, $\mathbf{O}(m)$ and $\mathbf{V}(m)$ can be obtained by using singular value decomposition of $\mathbf{H}(m)$. Using (8), (7) can be arranged as follows: 


$$
\begin{aligned}
e_{m} & =\mathbb{E}\left[\| \mathbf{s}(m)+\mathbf{U}^{H}(m) \mathbf{w}(m)\right. \\
& -\mathbf{C}_{B B}^{H}(m) \mathbf{C}_{R F}^{H} \mathbf{H}(m) \mathbf{P}_{R F} \mathbf{P}_{B B}(m) \mathbf{s}(m) \\
& \left.-\mathbf{C}_{B B}^{H}(m) \mathbf{C}_{R F}^{H} \mathbf{w}(m) \|_{F}^{2}\right] \\
& =\mathbb{E}[\underbrace{\left[\mathbf{I}-\mathbf{C}_{B B}^{H}(m) \mathbf{C}_{R F}^{H} \mathbf{H}(m) \mathbf{P}_{R F} \mathbf{P}_{B B}(m)\right) \mathbf{s}(m)}_{\text {signal part }, e_{m}^{s}} \\
& +\underbrace{\left(\mathbf{U}^{H}(m)-\mathbf{C}_{B B}^{H}(m) \mathbf{C}_{R F}^{H}\right) \mathbf{w}(m)}_{\text {noise part } e_{m}^{n}} \|_{F}^{2}] .
\end{aligned}
$$

(9) can be directly optimized to obtain exact solution as follows:

$$
\min _{\mathbf{C}_{R F}, \mathbf{C}_{B B}(m), \mathbf{P}_{R F}, \mathbf{P}_{B B}(m)} \sum_{m} \mathbb{E}\left[e_{m}\right] \text {, }
$$

or can be first partitioned into two parts like as signal parts and noise part, and then each part can be separately optimized to obtain a suboptimal solution. Since the aim of this work is to design a suboptimal hybrid beamformer, we have made a separate optimization of each of the signal part and the noise part. Those problems in either case are still non-convex. Each of two optimization problem can be solved by a single singular value decomposition over concatenated matrices. Let's define those concatenated matrices as

$$
\begin{aligned}
& \overline{\mathbf{P}}_{o p t}=\left[\mathbf{P}_{o p t}(1) \mathbf{P}_{o p t}(2) \ldots \mathbf{P}_{o p t}(M)\right] \in C^{N_{B S} \times M N_{S}}, \\
& \overline{\mathbf{P}}_{B B}=\left[\mathbf{P}_{B B}(1) \mathbf{P}_{B B}(2) \ldots \mathbf{P}_{B B}(M)\right] \in C^{N_{R F} \times M N_{S}}, \\
& \overline{\mathbf{C}}_{o p t}=\left[\mathbf{C}_{o p t}(1) \mathbf{C}_{o p t}(2) \ldots \mathbf{C}_{o p t}(M)\right] \in C^{N_{M S} \times M N_{S}}, \\
& \overline{\mathbf{C}}_{B B}=\left[\mathbf{C}_{B B}(1) \mathbf{C}_{B B}(2) \ldots \mathbf{C}_{B B}(M)\right] \in C^{N_{R F} \times M N_{S}} .
\end{aligned}
$$

We can now define final form of the considered problem as

$$
\begin{gathered}
\min _{\mathbf{C}_{R F}, \overline{\mathbf{C}}_{B B}} \sigma_{w}^{2} \operatorname{Tr}\left[\left(\mathbf{C}_{o p t}^{H}-\overline{\mathbf{C}}_{B B}^{H} \mathbf{C}_{R F}^{H}\right)\left(\mathbf{C}_{\text {opt }}^{H}-\overline{\mathbf{C}}_{B B}^{H} \mathbf{C}_{R F}^{H}\right)^{H}\right] \\
\text { s.t. } \mathbf{C}_{R F} \in \mathcal{C}_{R F} \\
\min _{\mathbf{P}_{R F}, \overline{\mathbf{P}}_{B B}} \frac{P_{t}}{M N_{S}} \operatorname{Tr}\left[\left(\mathbf{I}-\overline{\overline{\mathbf{C}}}_{B B}^{H} \widehat{\mathbf{C}}_{R F}^{H} \mathbf{U} \boldsymbol{\Sigma} \mathbf{V}^{H} \mathbf{P}_{R F} \overline{\mathbf{P}}_{B B}\right)\right. \\
\left.\left(\mathbf{I}-\hat{\overline{\mathbf{C}}}_{B B}^{H} \widehat{\mathbf{C}}_{R F}^{H} \mathbf{U} \boldsymbol{\Sigma} \mathbf{V}^{H} \mathbf{P}_{R F} \overline{\mathbf{P}}_{B B}\right)^{H}\right] \\
\text { s.t. } \mathbf{P}_{R F} \in \mathbf{P}_{R F}
\end{gathered}
$$

\begin{tabular}{|c|c|}
\hline Step 1 & Initialize \\
\hline & $\mathbf{C}_{r e s}=\overline{\mathbf{C}}_{o p t}=\mathbf{U}$ \\
\hline & $\mathbf{C}_{R F}=[]$ \\
\hline \multirow[t]{6}{*}{ Step 2} & while $i \leq N_{R F}$ do \\
\hline & (2.1) $\Xi_{C}=\mathbf{A}_{a}^{H} \mathbf{C}_{r e s}$ \\
\hline & (2.2) $k=\underset{j}{\arg \max }\left[\Xi_{C}^{H} \Xi_{C}\right]_{j, j}$ \\
\hline & (2.3) $\mathbf{C}_{R F}=\left[\mathbf{C}_{R F} \mid \mathbf{A}_{a}(:, k)\right]$ \\
\hline & (2.4) $\overline{\overline{\mathbf{C}}}_{B B}=\left(\mathbf{C}_{R F}^{H} \mathbf{C}_{R F}\right)^{-1} \overline{\mathbf{C}}_{o p t}$ \\
\hline & (2.5) $\mathbf{C}_{r e s}=\frac{\overline{\mathbf{C}}_{o p t}-\mathbf{C}_{R F} \overline{\overline{\mathbf{C}}}_{B B}}{\left\|\overline{\mathbf{C}}_{o p t}-\mathbf{C}_{R F} \overline{\mathbf{C}}_{B B}\right\|_{F}}$ \\
\hline Step 3 & Normalize $\overline{\overline{\mathbf{C}}}_{B B} \quad \overline{\overline{\mathbf{C}}}_{B B}=\frac{\overline{\overline{\mathbf{C}}}}{\| \mathrm{C}_{R F}}$ \\
\hline
\end{tabular}

\begin{tabular}{|c|c|}
\hline \multirow[t]{6}{*}{ Step 2} & while $k \leq N_{R F}$ do \\
\hline & (2.1) $\Xi_{P}=\mathbf{A}_{d}^{H} \mathbf{P}_{\text {res }}$ \\
\hline & (2.2) $k=\underset{i}{\arg \max }\left[\boldsymbol{\Xi}_{P}^{H} \boldsymbol{\Xi}_{P}\right]_{j,}$ \\
\hline & (2.3) $\mathbf{P}_{R F}=\left[\mathbf{P}_{R F} \mid \mathbf{A}_{d}(:, k)\right]$ \\
\hline & (2.4) $\overline{\overline{\mathbf{P}}}_{B B}=\left(\mathbf{P}_{R F}^{H} \mathbf{P}_{R F}\right)^{-1} \overline{\mathbf{P}}_{o p t} \wedge$ \\
\hline & (2.5) $\mathbf{P}_{r e s}=\frac{\overline{\mathbf{P}}_{o p t}-\mathbf{P}_{R F} \hat{\overline{\mathbf{P}}}_{B B}}{\left\|\overline{\mathbf{P}}_{o p t}-\mathbf{P}_{R F} \overline{\bar{P}}_{B B}\right\|_{F}}$ \\
\hline Step 3 & Normalize $\overline{\overline{\mathbf{P}}}_{B B}, \overline{\overline{\mathbf{P}}}_{B B}=\sqrt{\frac{I}{M}}$ \\
\hline
\end{tabular}

where $\hat{\overline{\mathbf{C}}}_{B B}$ and $\widehat{\mathbf{C}}_{R F}$ are the matrices obtained from the solution of (12). Since this problem has a smilar structure as its single carrier part form in [28], it can be extended to multicarrier case and then it can be solved using Orthogonal Matching Pursuit (OMP) algorithm leveraged from the compressive sensing domain. The optimization procedure can be defined
Table 1. OMP Algorithms

\section{Algorithm for Eq. (12)}

\section{Algorithm for Eq. (13)}

\begin{tabular}{ll}
\hline Step 1 & Initialize \\
\hline & $\mathbf{P}_{\text {res }}=\overline{\mathbf{P}}_{\text {opt }}=\left(\overline{\overline{\mathbf{C}}}_{B B}^{H} \overline{\mathbf{C}}_{R F}^{H} \mathbf{U} \mathbf{\Sigma} \mathbf{V}^{H}\right)^{-1}$ \\
\hline $\mathbf{P}_{R F}=[]$
\end{tabular}

into two steps. In the first step, the noise part is optimized and suboptimal combiner matrices $\overline{\overline{\mathbf{C}}}_{R F}$ and $\overline{\overline{\mathbf{C}}}_{B B}$ are obtained. In the second step, these suboptimal combiner matrices are inserted into the signal part, and then the signal part is optimized to obtain suboptimal precoder matrices $\widehat{\overline{\mathbf{P}}}_{R F}$ and $\overline{\overline{\mathbf{P}}}_{B B}$. Spesifically, we apply the OMP algorithms in Table 1 for both two steps to obtain suboptimal solution of the considered problem. 


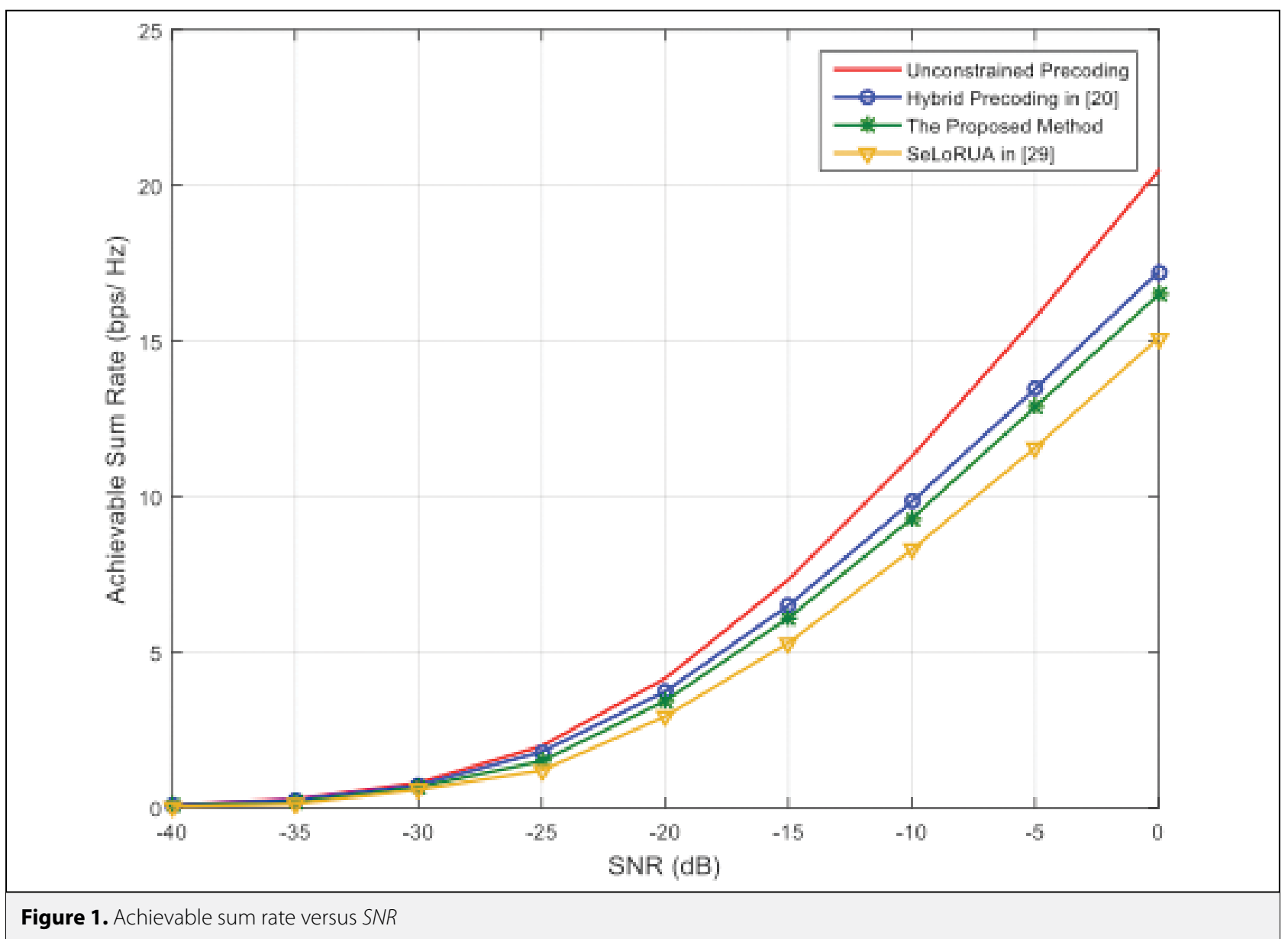

\section{Computer Simulations}

In this section, we present computer simulation results to reveal the performance of the proposed transceiver which consists of hybrid precoder and combiner. It is assumed a $\mathrm{mmW}$ system operating at $28 \mathrm{GHz}$ where transmitter $\mathrm{BS}$ and receiver MS have multiple antennas and limited number of RF chains. BS and MS are equipped with Uniform Linear Array (ULA), number of antennas in each of which are $N_{B S}=64$ and $N_{M S}=16$ respectively with $N_{R F}=3$. The AoA and the AoD are assumed to be uniformly distributed in $[0,2 \pi]$ with angle spread of $10^{\circ}$ for ULAs. The number of clusters for the $\mathrm{mmW}$ channel is $L=8$ , and each cluster has $R_{l}=7$ rays and unity average power. Complex path gain of the channel $\alpha_{r_{1}}$ are i.i.d. and follow the complex distribution $\mathcal{N}\left(0, \sigma_{r_{1}}^{2}\right)$. The number of subcarriers is $M=512$, and the cyclic prefix length is 128 . All the simulation results are generated by averaging 2000 randomly chosen channel realizations.

In Figure 1, the performance of the proposed suboptimal method is compared with different schemes. In this simulation, The number of transmitted streams is fixed at $N_{S}=3$. As can be seen from this figure, the suboptimal method proposed in this study exhibits a better performance than the method pro- posed in [29], but shows almost the same performance with the method proposed in [20]. This performance is quite good for a suboptimal method.

Figure 2 shows the comparison of the performance of the proposed suboptimal algorithm with the digital unconstrained solution for different numbers of transmission data streams. First, the performance of both coding methods increases and then decreases. The reason for this decrease is the sparse structure of the $\mathrm{mmW}$ channel and allocating of power equally between the different streams. The solution to this problem is what is called multimodal pre-coding. In addition, this figure shows that the difference between the proposed suboptimal solution and the digital unconstrained solution is small when the number of data streams has both the small and the large values. This is why the $\mathrm{mmW}$ channels are sparse.

\section{Conclusion}

In this work, we have presented a suboptimal hybrid precoding and combining design for downlink of a multicarrier massive MIMO system. It has a simple implementation and relatively lower computational complexity since it partitiones the objec- 


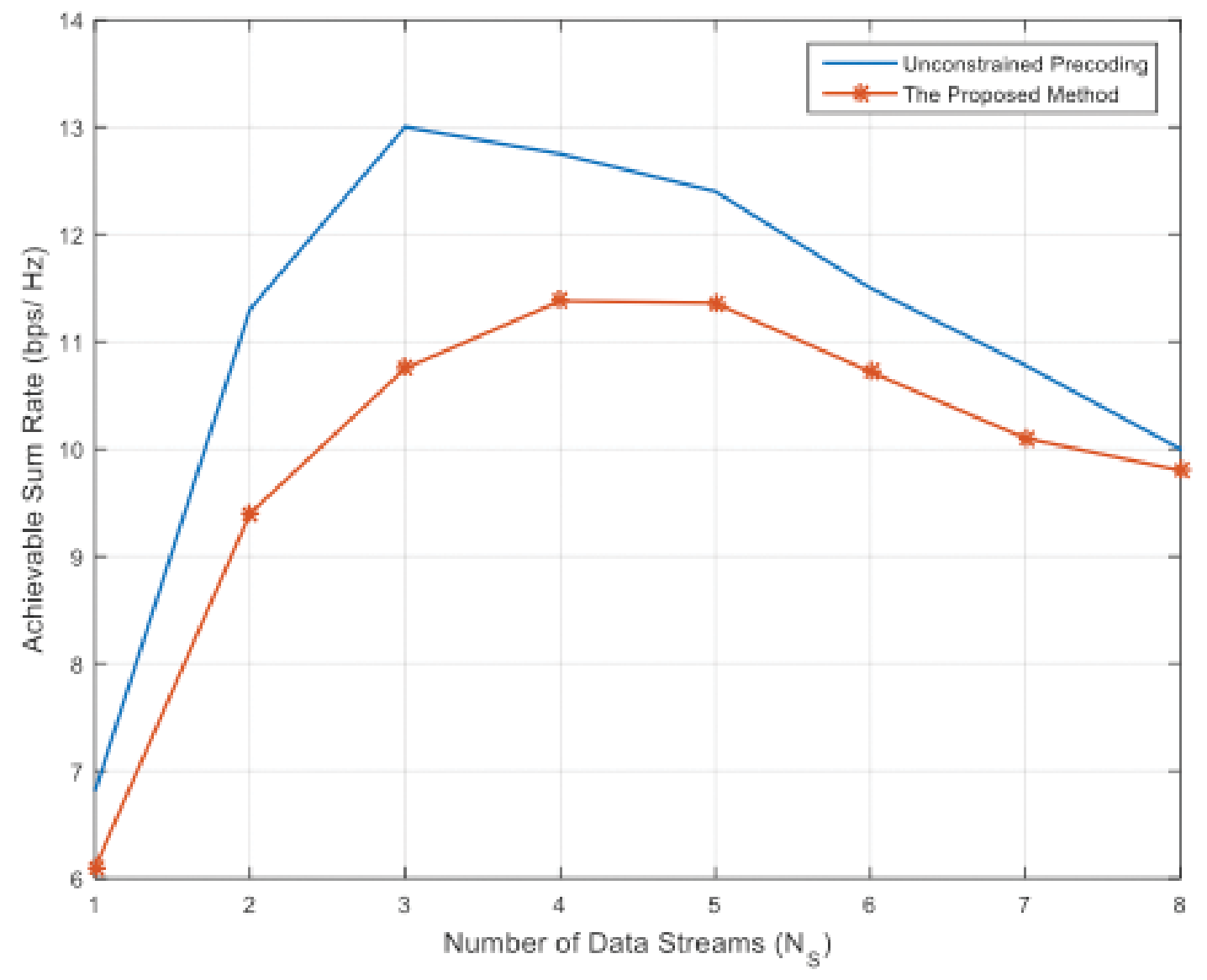

Figure 2. SER versus number of iterations for different $N_{S}$

tive function into two parts such as signal part and noise part. The reason for this is that the multi-parameter and multi-dimensional optimization problem is transformed into two parallel optimization problems with fewer parameters and fewer dimensions. The optimization procedure can be defined into two steps. In the first step, the noise part is optimized and suboptimal combiner matrices are obtained. In the second step, these suboptimal combiner matrices are inserted into the signal part, and then the signal part is optimized to obtain suboptimal precoder matrices. The performance of the proposed algorithm is sufficient. Simulation results show that it outperforms the precoding algorithm in [29], and has a close performance to the state of the art sparse precoding algorithm in [20].

\section{References}

1. A. Osseiran, V. Braun, T. Hidekazu, P. Marsch, H. Schotten, H. Tullberg, M. A. Uusitalo, M. "Schellman, The foundation of the mobile and wireless communications system for 2020 and beyond: Challenges, enablers and technology solutions". In: 2013 IEEE 77th Vehicular Technology Conference (VTC Spring), pp. 1-5, 2013.
2. IEEE standard for information technology- local and metropolitan area networks- specific requirements- part 15.3: Amendment 2: Millimeter-wave-based alternative physical layer extension. IEEE Std 802.15.3c-2009 (Amendment to IEEE Std 802.15.3-2003), 1-200, 2009.

3. Iso/iec/ieee international standard for information technology-telecommunications and information exchange between systems-local and metropolitan area networks-specific requirements-part 11: Wireless lan medium access control (mac) and physical layer (phy) specifications amendment 3: Enhancements for very high throughput in the $60 \mathrm{ghz}$ band (adoption of IEEE std 802.11ad-2012). ISO/IEC/IEEE 8802-11:2012/Amd.3:2014(E), 1-634, 2014.

4. Z. Pi, F. Khan, "An introduction to millimeter-wave mobile broadband systems," IEEE Communications Magazine, vol. 49, pp. 101107, 2011. [CrossRef]

5. M. R. Akdeniz, Y. Liu, M. K. Samimi, S. Sun, S. Rangan, T. S. Rappaport, E. Erkip, E, "Millimeter wave channel modeling and cellular capacity evaluation," IEEE Journal on Selected Areas in Communications, vol. 32, pp. 1164-1179, 2014. [CrossRef]

6. J. Wang, "Beam codebook based beamforming protocol for multi-gbps millimeter-wave wpan systems," IEEE Journal on Selected Areas in Communications, vol. 27, pp. 1390-1399, 2009. [CrossRef] 
7. S. Hur, T. Kim, D. J. Love, J. V. Krogmeier, T. A. Thomas, A. Ghosh, "Millimeter wave beamforming for wireless backhaul and access in small cell networks," IEEE Transactions on Communications vol. 61, pp. 4391-4403, 2013. [CrossRef]

8. Y. M. Tsang, A. S. Y. Poon, S. Addepalli, "Coding the beams: Improving beamforming training in mmwave communication system," In: 2011 IEEE Global Telecommunications Conference - GLOBECOM 2011, pp. 1-6, 2011. [CrossRef]

9. T. Kim, J. Park, J. Y. Seol, S. Jeong, J. Cho, W. Roh, "Tens of gbps support with mmwave beamforming systems for next generation communications," In: 2013 IEEE Global Communications Conference (GLOBECOM), pp. 3685-3690, 2013.

10. O. E. Ayach, S. Rajagopal, S. Abu-Surra, Z. Pi, R. W. Heath, "Spatially sparse precoding in millimeter wave mimo systems," IEEE Transactions on Wireless Communications, vol. 13, pp. 1499-1513, 2014. [CrossRef]

11. A. Alkhateeb, O. E. Ayach, G. Leus, R. W. Heath, “Hybrid precoding for millimeter wave cellular systems with partial channel knowledge," In: 2013 Information Theory and Applications Workshop (ITA), pp. 1-5, 2013. [CrossRef]

12. S. Sun, T. S. Rappaport, R. W. Heath, A. Nix, S. Rangan, "Mimo for millimeter-wave wireless communications: beamforming, spatial multiplexing, or both?" IEEE Communications Magazine, vol. 52, pp. 110-121, 2014. [CrossRef]

13. A. Alkhateeb, J. Mo, N. Gonzalez-Prelcic, R. W. Heath, "Mimo precoding and combining solutions for millimeter-wave systems," IEEE Communications Magazine, vol. 52, pp. 122-131, 2014. [CrossRef]

14. T. S. Rappaport, S. Sun, R. Mayzus, H. Zhao, Y. Azar, K. Wang, G. N. Wong, J. K. Schulz, M. Samimi, F. Gutierrez, "Millimeter wave mobile communications for $5 \mathrm{~g}$ cellular: It will work!", IEEE Access 1 , 335-349, 2013. [CrossRef]

15. P. Schniter, A. Sayeed, "Channel estimation and precoder design for millimeter-wave communications: The sparse way," In: 2014 48th Asilomar Conference on Signals, Systems and Computers, pp. 273-277, 2014. [CrossRef]

16. M. K. Samimi, T. S. Rappaport, "Ultra-wideband statistical channel model for non line of sight millimeter-wave urban channels," In: 2014 IEEE Global Communications Conference, pp. 3483-3489, 2014. [CrossRef]

17. C. E. Chen, "An iterative hybrid transceiver design algorithm for millimeter wave mimo systems," IEEE Wireless Communications Letters vol. 4, pp. 285-288, 2015. [CrossRef]

18. X. Yu, J. C. Shen, J. Zhang, K. B. Letaief, "Alternating minimization algorithms for hybrid precoding in millimeter wave mimo sys- tems," IEEE Journal of Selected Topics in Signal Processing vol. 10, 485-500, 2016. [CrossRef]

19. S. Park, R. W. Heath, "Frequency selective hybrid precoding in millimeter wave ofdma systems," In: 2015 IEEE Global Communications Conference (GLOBECOM), pp. 1-6, 2015. [CrossRef]

20. A. Alkhateeb, R. W. Heath, "Frequency selective hybrid precoding for limited feedback millimeter wave systems," IEEE Transactions on Communications, vol. 64, pp. 1801-1818, 2016. [CrossRef]

21. A. Saleh, R. Valenzuela, "A statistical model for indoor multipath propagation," IEEE Journal on Selected Areas in. Communications. (2006).

22. W. U. Bajwa, J. Haupt, A. M. Sayeed, R. Nowak, "Compressed channel sensing: A new approach to estimating sparse multipath channels," Proceedings of the IEEE, vol. 98, pp. 1058-1076, 2010. [CrossRef]

23. L. Liang, W. Xu, X. Dong, "Limited feedback-based multi-antenna relay broadcast channels with block diagonalization," IEEE Transactions on Wireless Communications, vol. 12, pp. 4092-4101, 2013. [CrossRef]

24. X. Xue, T. E. Bogale, X. Wang, Y. Wang, L. B. Le, “Hybrid analog-digital beamforming for multiuser mimo millimeter wave relay systems," In: 2015 IEEE/CIC International Conference on Communications in China (ICCC), pp. 1-7, 2015. [CrossRef]

25. G. Kwon, H. Park, "An efficient hybrid beamforming scheme for sparse millimeter wave channel," In: 2015 IEEE Global Communications Conference (GLOBECOM), pp. 1-6, 2015. [CrossRef]

26. N. Song, H. Sun, T. Yang, "Coordinated hybrid beamforming for millimeter wave multi-user massive mimo systems," In: 2016 IEEE Global Communications Conference (GLOBECOM), pp. 1-6, 2016. [CrossRef]

27. R. Rajashekar, L. Hanzo, "Iterative matrix decomposition aided block diagonalization for mm-wave multiuser mimo systems," IEEE Transactions on Wireless Communications, vol. 16, pp. 1372-1384, 2017. [CrossRef]

28. A. Alkhateeb, O. El Ayach, G. Leus, R. W. Heath, "Channel Estimation and Hybrid Precoding for Millimeter Wave Cellular Systems," IEEE Journal of Selected Topics in Signal Processing, vol. 8, pp. 831-846, 2014. [CrossRef]

29. J. Zhang, A. Wiesel and M. Haardt, "Low rank approximation based hybrid precoding schemes for multi-carrier single-user massive MIMO systems," IEEE International Conference on Acoustics, Speech and Signal Processing (ICASSP), Shanghai, pp. 3281-3285, 2016, [CrossRef] Ph.D. degree in electronics and telecommunication engineering from İstanbul Technical University, İstanbul, Turkey, in 1997, 2000, 2005 respectively. After working in different positions at İstanbul University, The Scientific and Technological Research Council of Turkey, Kadir Has University and Maltepe University, he joined to İstanbul Medeniyet University as an associate professor in 2015. His general research interests cover estimation theory, statistical signal processing, communication theory, wireless sensor networks and array processing. His current research areas are focused on wireless communication concepts with specific attention to channel estimation for spread-spectrum and multicarrier systems, on beamforming design for next generation systems and on optimal power allocation in wireless sensor networks. 1 